\title{
NOTE ON THE THEORY OF HEAT CONDUCTION
}

\author{
By M. S. Van Dusen
}

ABSTRACT

A simple transformation of the general equation for heat flow is given, which does not appear to be generally known. By means of this transformation most problems in steady heat flow with conductivity depending on temperature can be derived directly from the solutions of the same problems where the conductivity is assumed constant. The paper is entirely mathematical.

The general equation of heat flow by conduction is

$$
\operatorname{div}(k \nabla \theta)=c \frac{\partial \theta}{\partial t}
$$

where $\theta$ is the temperature at any point at the time $t$, and $k$ and $c$ are, respectively, the thermal conductivity and the heat capacity per unit volume of the medium at the temperature $\theta$ and at the point in question.

If $k$ is assumed to be independent of temperature, then for an isotropic medium. equation (1) reduces to the ordinary form, viz:

$$
\frac{k}{c} \nabla^{2} \theta=\frac{\partial \theta}{\partial t}
$$

The various treatises dealing wholly or in part with the mathematical theory of heat conduction invariably assume that both $k$ and $c$ are independent of temperature, and imply that otherwise the mathematical difficulties become very great. Carslaw ${ }^{1}$ states that in some mathematical investigations the conductivity, $k$, is assumed to be a linear function of temperature, but does not give specific references.

It will be shown, however, that in the majority of instances the variation of $k$ with temperature offers less difficulty than the variation of $c$ with temperature. If the conductivity $k$ is a known function of temperature, given by

$$
k=f(\theta)
$$

and if we introduce a new variable, $u$, definea as

$$
u \equiv \int_{\theta_{0}}^{\theta} f(\beta) d \beta
$$

then, by using the relations:

and

$$
\nabla u=k \nabla \theta
$$

$$
\frac{\partial u}{\partial t}=k \frac{\partial \theta}{\partial t}
$$


derived from (4), the general equation (1) reduces to

$$
\frac{k}{c} \nabla^{2} u=\frac{\partial u}{\partial t}
$$

$\nabla u$ is the (negative) heat flux, and in equation (4) the lower limit of the integral is arbitrary. It might be conveniently taken as zero, for then when $\theta=0, u=0$.

If in equation (5) the quantity $\frac{k}{c}$, termed the diffusivity, or the thermometric conductivity, is independent of temperature, equation (5) is identical in form with equation (2). This quantity, however, is not, in general, constant; but it may be remarked that for noncrystalline poorly conducting solids the diffusivity is more nearly independent of temperature than either $k$ or $c$ individually, which is assumed in the classical solutions of problems in heat flow. For such materials, therefore, equation (5) with $\frac{k}{c}$ constant is a better approximation than the classical form equation (2). At any rate the physical properties of the medium in question have been combined into one variable coefficient without loss of generality.

In all problems of steady heat flow, however, the time rates of change of both $\theta$ and $u$ vanish, the diffusivity consequently disappears, and equation (5) becomes

$$
\nabla^{2} u=0
$$

which is identical in form with the classical equation for steady heat flow. It follows, therefore, that if the boundary conditions are expressed in terms of $u$ instead of $\theta$, by means of equation (4), problems of steady heat flow with variable conductivity can be attacked by the same general methods that are used in the solution of problems with constant conductivity. In fact, in all cases where the boundary conditions are originally given in terms of temperature, normal heat flux, or a combination of both, solutions for variable conductivity are immediately derivable from the solutions of the same problems with constant conductivity. The general nature of this principle has apparently been overlooked, or at least not generally recognized.

If the boundary conditions are given in terms of differential equations, as in the case of heat transfer between a boundary surface and a fluid medium according to some experimental law of heat transfer, such as Newton's law, the solutions for $u$ do not follow directly from the solutions for $\theta$, for then the differential equations in terms of $u$ become different in form from those in terms of $\theta$. For example, it may be possible to find a solution of the equation: $\nabla^{2} \theta=0$, satisfying certain boundary conditions, among which is included:

$$
k \frac{\partial \theta}{\partial n}+h \theta=0
$$

over some portion of the boundary.

In terms of $u$, we must satisfy

and

$$
\nabla^{2} u=0 \text { in the solid }
$$

$$
\frac{\partial u}{\partial n}+h F(u)=0
$$


over the same portion of the boundary, the function $F(u)=\theta$ being derived from equation (4).

It is evident that equations (11) and (10) are different in form, and the solution in $u$ can not be derived directly from the solution in $\theta$.

As an example of the use of equation (6), let us consider the solution of a problem which was useful in connection with the design of apparatus for measuring the thermal conductivity of metals.

One end $(z=0)$ of a cylinder of length $c$ and radius $a$ is maintained at zero temperature, the other end $(z=c)$ being insulated. Heat is added at a constant rate to a portion of the convex suriace of length $b$ adjacent to the end $z=c$, the rest of the convex surface being insulated. Required: The temperature distribution in the cylinder when the steady state has been attained.

\section{CASE I.-Constant thermal conductivity}

We have to solve: $\nabla^{2} \theta=0$, or in cylindrical coordinates:

$$
\frac{\partial^{2} \theta}{\partial r^{2}}+\frac{1}{r} \frac{\partial \theta}{\partial r}+\frac{\partial^{2} \theta}{\partial z^{2}}=0
$$

subject to the boundary conditions:

When

$$
\begin{aligned}
& z=0, \theta=0 \\
& z=c, \frac{\partial \theta}{\partial z}=0 \\
& 0<z<c-b), \frac{\partial \theta}{\partial r}=0 \\
& \left.\begin{array}{r}
r=a \\
c-b<z<c
\end{array}\right\}, \frac{\partial \theta}{\partial r}=\frac{h}{k}
\end{aligned}
$$

where $h$ is the inward heat flux per unit area normal to the convex surface, and $k$ the thermal conductivity of the material.

By considering the image of the cylinder extending from the end $z=c$ to $z=2 c$, maintaining the imaginary end at zero temperature, and adding heat to the portion of the convex surface extending from $z=c$ to $z=b+c$, it is evident from symmetry that no heat will flow across the surface $z=c$, and conditions in the actual cylinder will be unchanged. By this device the solution is readily built up from known particular solutions of equation (7), and the final result is as follows:

$$
\theta=\frac{8 h c}{k \pi^{2}} \sum_{n=1}^{n \rightarrow \infty} \frac{\sin \frac{n \pi}{2} \sin \frac{n \pi b}{2 c}}{-i n^{2} J_{1}\left(i \frac{n \pi a}{2 c}\right)} \sin \frac{n \pi z}{2 c} J_{0}\left(i \frac{n \pi r}{2 c}\right)
$$

where $i$ is the usual symbol for $\sqrt{-1}$, and $J_{0}$ and $J_{1}$ are, respectively, Bessel's functions of orders 0 and 1 . 
CASE II.-Variable thermal conductivity

Let $k=k_{0}(1+\alpha \theta)$; therefore $u=k_{0}\left(\theta+\frac{\alpha}{2} \theta^{2}\right)$. We have to solve:

$$
\nabla^{2} u=0
$$

the boundary conditions in $u$ being:

When

$$
\begin{aligned}
& z=0, u=0 \\
& z=c, \frac{\partial u}{\partial z}=0 \\
& \left.\begin{array}{r}
r=a \\
0<z<c-b
\end{array}\right\}, \quad \frac{\partial u}{\partial r}=0 \\
& \left.\begin{array}{r}
r=a \\
c-b<z<c
\end{array}\right\}, \quad \frac{\partial u}{\partial r}=h
\end{aligned}
$$

The solution for $u$ is evidently the same as equation (8) except that $\theta$ is replaced by $u$, and $\frac{h}{k}$ by $h$ alone. Substituting for $u$ its value in terms of $\theta$, we have finally

$$
\theta+\frac{\alpha}{2} \theta^{2}=\frac{8 h c}{k_{0} \pi^{2}} \sum_{n=1}^{n \rightarrow \infty} \frac{\sin \frac{n \pi}{2} \sin \frac{n \pi b}{2 c}}{-i n^{2} J_{1}\left(i \frac{n \pi a}{2 c}\right)} \sin \frac{n \pi z}{2 c} J_{0}\left(i \frac{n \pi r}{2 c}\right)
$$

This equation can, of course, be solved explicitly for $\theta$, or numerical calculations can be made by successive approximations, since the value of the cofficient $\alpha$ is ordinarily rather small. Evidently, if $\alpha=0$, equation (9) must reduce to equation (8), for then the conductivity is constant and equal to $k_{0}$.

Washington, February 5, 1930. 